\title{
An accessible method for screening aerosol filtration identifies poor-performing commercial masks and respirators
}

\author{
Katherine Schilling ${ }^{1}$ - Drew R. Gentner $\mathbb{B}^{1,2} \cdot$ Lawrence Wilen $^{3} \cdot$ Antonio Medina $^{3} \cdot$ Colby Buehler $^{1,2}$. \\ Luis J. Perez-Lorenzo ${ }^{4} \cdot$ Krystal J. Godri Pollitt $^{1,5} \cdot$ Reza Bergemann $^{3} \cdot$ Nick Bernardo $^{3} \cdot$ Jordan Peccia $^{1}$. \\ Vincent Wilczynski ${ }^{3} \cdot$ Lisa Lattanza $^{6}$
}

Received: 5 May 2020 / Revised: 10 July 2020 / Accepted: 27 July 2020 / Published online: 6 August 2020

(c) The Author(s), under exclusive licence to Springer Nature America, Inc. 2020

\begin{abstract}
Background The COVID-19 pandemic has presented an acute shortage of regulation-tested masks. Many of the alternatives available to hospitals have not been certified, leaving uncertainty about their ability to properly protect healthcare workers from SARS-CoV-2 transmission.

Objective For situations where regulatory methods are not accessible, we present experimental methods to evaluate mask filtration and breathability quickly via cost-effective approaches (e.g., $\sim 2000$ USD) that could be replicated in communities of need without extensive infrastructure. We demonstrate the need for screening by evaluating an existing diverse inventory of masks/respirators from a local hospital.

Methods Two experimental approaches are presented to examine both aerosol filtration and flow impedance (i.e., breathability). For one of the approaches ("quick assessment"), screening for appropriate filtration could be performed under 10 min per mask, on average. Mask fit tests were conducted in tandem but are not the focus of this study.

Results Tests conducted of 47 nonregulation masks reveal variable performance. A number of commercially available masks in hospital inventories perform similarly to N95 masks for aerosol filtration of $0.2 \mu \mathrm{m}$ and above, but there is a range of masks with relatively lower filtration efficiencies (e.g., <90\%) and a subset with poorer filtration (e.g., <70\%). All masks functioned acceptably for breathability, and impedance was not correlated with filtration efficiency.

Significance With simplified tests, organizations with mask/respirator shortages and uncertain inventories can make informed decisions about use and procurement.
\end{abstract}

Keywords Personal protective equipment $(\mathrm{PPE}) \cdot$ Masks $\cdot$ N95 respirators $\cdot$ COVID-19 $\cdot$ SARS-CoV-2

These authors contributed equally: Katherine Schilling, Drew R. Gentner, Lawrence Wilen

Supplementary information The online version of this article (https:// doi.org/10.1038/s41370-020-0258-7) contains supplementary material, which is available to authorized users.

Lisa Lattanza

lisa.lattanza@yale.edu

1 Department of Chemical and Environmental Engineering, School of Engineering and Applied Science, Yale University, New Haven, CT 06511, USA

2 SEARCH (Solutions for Energy, Air, Climate and Health) Center, Yale University, New Haven, CT, USA

3 School of Engineering and Applied Science, Yale University,

\section{Introduction}

The COVID-19 pandemic has presented an acute need for masks and respirators to be used by healthcare workers on the frontlines and growing needs for essential workers and the public to wear masks in affected areas. The rapid shortage of medical N95 respirators or other certified masks creates an urgent demand for suitable alternatives. This has

New Haven, CT 06511, USA

4 Department of Mechanical Engineering, School of Engineering and Applied Science, Yale University, New Haven, CT 06511, USA

5 Department of Environmental Health Sciences, School of Public Health, Yale University, New Haven, CT 06510, USA

6 Department of Orthopaedics and Rehabilitation, School of Medicine, Yale University, New Haven, CT 06511, USA 
led to an influx of a wide variety of masks that have not been tested by the National Institute for Occupational Health and Safety (NIOSH) into the medical community and the public with little to no assurance of performance. Hospitals are often faced with deciding which other type of masks (e.g., KN95) to consider buying when the traditional supplies of tested masks are no longer available for purchase [1]. Many claim to have filtration characteristics equivalent to traditional $\mathrm{N} 95$ masks but have not undergone the same testing for N95 masks in the U.S. There is also a large influx of counterfeit masks into the market due to mask shortages during the pandemic [2-4]. Many hospitals are receiving donations from a well-intentioned public that range from non-medical respirators and surgical masks to handsewn facemasks. Most of these donated items have not been confirmed by NIOSH to provide comparable protection from the transmission of SARS-CoV-2 to a standard medical N95 mask [5]. As such, the decision to use them without testing information poses significant risk to healthcare and other essential workers.

Currently the certification capacity of NIOSH is too limited to accommodate the high demand for testing of masks and allow for timely decision making on purchases or the use of donated masks during this critical nationwide shortage. The pandemic and need for accessible mask testing methods are also likely to reach regions with decreased capacity for mask testing (e.g., developing nations). In the absence of available regulation testing facilities or the specific and costly equipment to replicate regulatory methods, we present a screening method to quickly evaluate masks using an accessible approach that could be replicated in communities lacking the infrastructure necessary for regulation tests. These methods are not intended to replace regulation approaches, but to provide alternatives to nonexperts in times of need to screen and prioritize the use or acquisition of masks/respirators and to complement the existing body of literature on mask and alternative mask testing (e.g., [6]). As such, readily accessible equipment has been used to maintain accessibility for a greater diversity of communities.

There are several key considerations for mask performance: (a) filtration, (b) flow impedance (i.e., breathability), (c) fit, and (d) continued performance under environmental conditions (e.g., wetting). The assessments performed in this document focuses on the first two aspects. In tandem with this study, masks were also evaluated for fit using a commercial leak detection apparatus, conducted by members of Yale University's Office of Environmental Health and Safety, to characterize and reduce penetration of aerosols at the edges of the masks [7]. In order for a mask to be deemed appropriate for clinical use in a COVID-19 patient setting it must pass the filtration and breathability tests as well as a fit test. NIOSH, FDA, and other federal methods for medical testing masks and respirators have differences in test aerosols (e.g., $\mathrm{NaCl}$ solution vs. latex spheres) and measurement methods [8-12] (e.g., offline gravimetric vs. online counters) as summarized and compared in detail in Rengasamy et al. [5].

Respiratory droplets and aerosols are emitted from humans during coughing, sneezing, breathing, talking, or intubation that could contain viruses, including SARS-CoV2. Exhaled aerosol/droplets may span from the nominal size of SARS-CoV-2 ( 120 nm) up to $10 \mu \mathrm{m}$ or larger [13-19], and can decrease in size with evaporation of condensed water [20-22]. The World Health Organization identifies two main categories of particles as key factors in coronavirus transmission: "respiratory droplets" $(>5-10 \mu \mathrm{m}$ in diameter) and "droplet nuclei" (aerosols $<5 \mu \mathrm{m}$ in diameter) [23]; the latter of which includes the typical test aerosol diameters for U.S. agencies involved in mask certification $(0.075-5 \mu \mathrm{m})$ [5]. The airborne lifetime of these humangenerated aerosol/droplets are size-dependent, reaching upwards of several hours for aerosols in the $0.1-1-\mu \mathrm{m}$ size range $[24,25]$ and suspended SARS-CoV-2 can survive airborne for over $1 \mathrm{~h}$ at moderate humidities $(\mathrm{RH}=65 \%)$ [26]. Aerosols in that size range containing SARS-CoV-2 and surface contamination have been observed in staff areas of hospitals away from patients [18, 19, 27]. Therefore, it is important to consider a wide range of droplet and aerosol sizes for mask filtration efficacy.

Our overall goals are (a) to disseminate simplified testing setups that can be used in comparative evaluations of nonregulation or alternative masks against regulation masks and (b) present results from our survey of a set of commercially available masks representative of those entering U.S. hospital networks. Testing specifically focuses on flow impedance and aerosol filtration, both of which are evaluated in the traditional NIOSH mask certification process. This study utilizes more readily available equipment and resources to conduct similar assessments in an experimentally comparable procedure, but does not attempt to replicate or claim NIOSH approval. Its purpose is to allow health professionals to make informed decisions on the most appropriate masks to use when trusted PPE is not available.

\section{Experimental setup}

\section{Filtration testing overview}

With the intent of evaluating filtration efficiency without purporting to replicate NIOSH equivalency, the methods designed in this study are aimed at testing masks with relevant, reproducible aerosol distributions at face velocities appropriate for human respiration. To evaluate performance, we utilize a combustion-generated polydisperse 


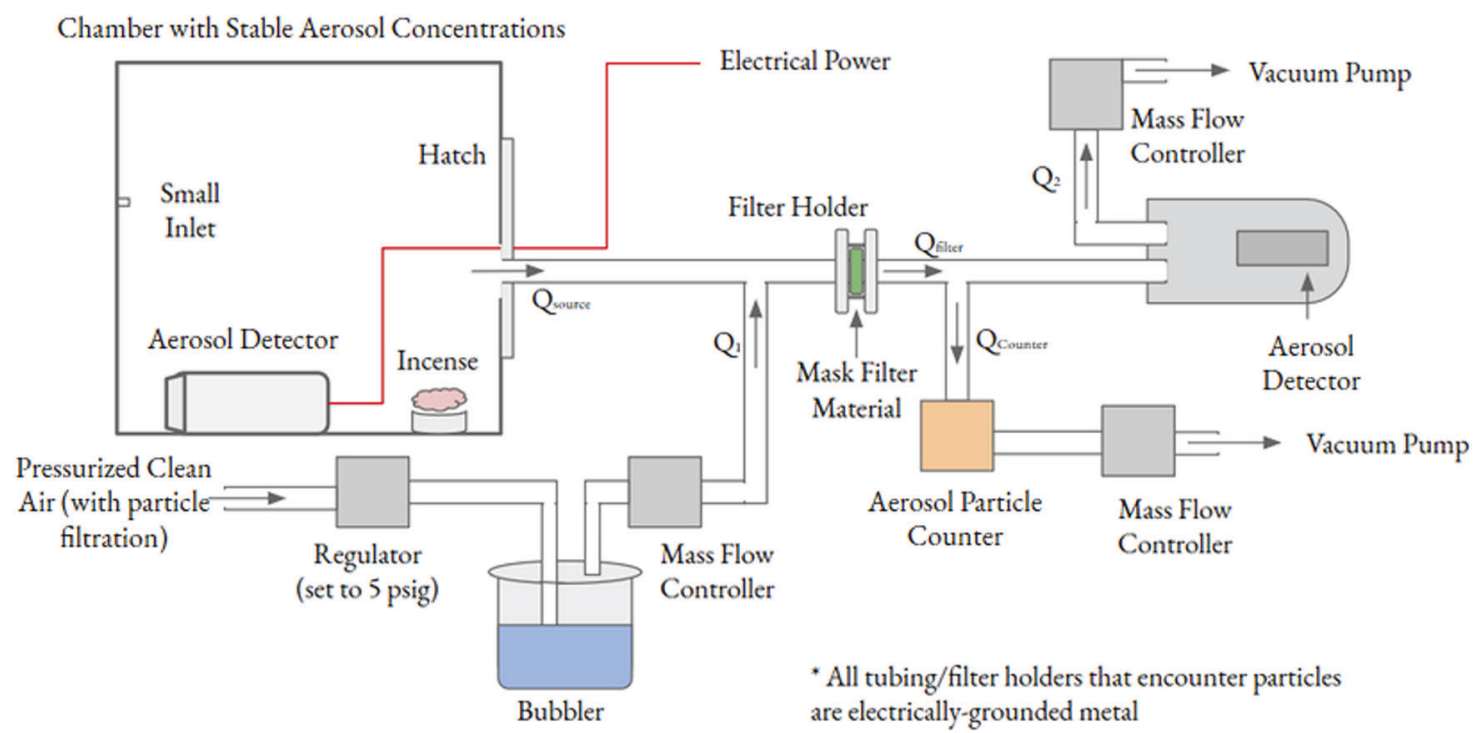

Fig. 1 Primary experimental setup for aerosol filtration assessmen. "Aerosol detectors" refer to SEARCH monitors and the "aerosol particle counter" is the AirNet instrument (in orange).

aerosol and measure removal efficiencies for several size ranges. Using readily available aerosol instrumentation, size-resolved aerosol number, and mass concentrations are measured upstream and downstream of a test mask material. The measured aerosol removal efficiencies of untested respirators and masks are then compared with those of a production lot of regulatory N95 masks to establish performance criteria.

The combustion-generated aerosol is produced via incense inside a sealed $0.25-\mathrm{m}^{3}$ acrylic box that serves as a contained aerosol source (Fig. 1). To achieve the desired aerosol concentrations (e.g., $50-100 \mu \mathrm{g} \mathrm{m}^{-3}$ ), a stick of burning incense is inserted briefly via a small port in the source chamber. As the incense smolders, it generates humidity and organic compounds, which condense to create a polydisperse aerosol that comprises a wide range of sizes, across the range of concern [28-30], including both aerosol and droplet emissions from humans (e.g., Fig. S14). Sufficient time is allowed for the aerosol population to become well-mixed and stable in the chamber, using a real-time sensor to track size-resolved concentrations.

The aerosols produced in the source chamber may be too highly concentrated depending on the measurement instrumentation used (e.g., the AirNet aerosol particle counter used in this study, described in detail below). Thus, the flow from the chamber is diluted using pressurized house air that is humidified via a bubbler and regulated by a mass flow controller (AliCat) to control the rate of dilution, though other flow control options are feasible. This diluted aerosol stream is used to test the mask material, which is a disk cut from a full mask using a die for consistency (Fig. S3). The disk is housed in a filter holder, in this case a custom aluminum holder with an exposed filter area of $30.5 \mathrm{~mm}$ in diameter (Fig. S1), but commercially available filter holders should suffice, and disks may be cut by hand. Grounded metal tubing was used leading to the filter holder and the detectors to reduce losses of charged particles. However, charge neutralization of aerosols is not employed in this setup, and aerosol charge may play a role in filtration for some masks.

This test method, as with standard test methods, mimics the face velocity (equal to the volume flow rate/surface area) of aerosol deposition on a mask during typical human inspiratory breathing flow rates of 65-220 LPM [5] corresponding to face velocities of $\sim 6.4-21.7 \mathrm{~cm} / \mathrm{s}$ for masks ranging in area from $130-225 \mathrm{~cm}^{2}$ (e.g., Table S1). These face velocities are achieved with our 30.5-mm disk sample using volume flow rates from 2.8 to $9.5 \mathrm{LPM}$ and most of the testing focused on $4.5 \mathrm{LPM}$ corresponding to $10 \mathrm{~cm} / \mathrm{s}$.

Two real-time detectors were used in the study, an AirNet (model 210, Particle Measurement Systems) and a SEARCH multipollutant monitor equipped with a Plantower A003 sensor from the "Solutions for Energy, AiR, Climate, and Health" Center at Yale-Johns Hopkins [31]. Other detectors may be used, provided the careful considerations detailed in this paper are followed. The AirNet detector measures the number concentration of aerosols in size bins of $0.2-0.3,0.3-0.5,0.5-1$, and $>1 \mu \mathrm{m}$. The SEARCH monitor measures mass and number concentrations of aerosols in size bins $0.3-0.5,0.5-1,1-2.5,2.5-5$, and $2.5-10 \mu \mathrm{m}$ with a lower sensitivity compared with the AirNet [31].

Upstream of the filter, the SEARCH detector is used to monitor and maintain reproducible and stable test aerosol concentrations in the source chamber. Downstream of the filter, aerosol concentrations were measured with both the AirNet and a second SEARCH instrument for redundancy. 


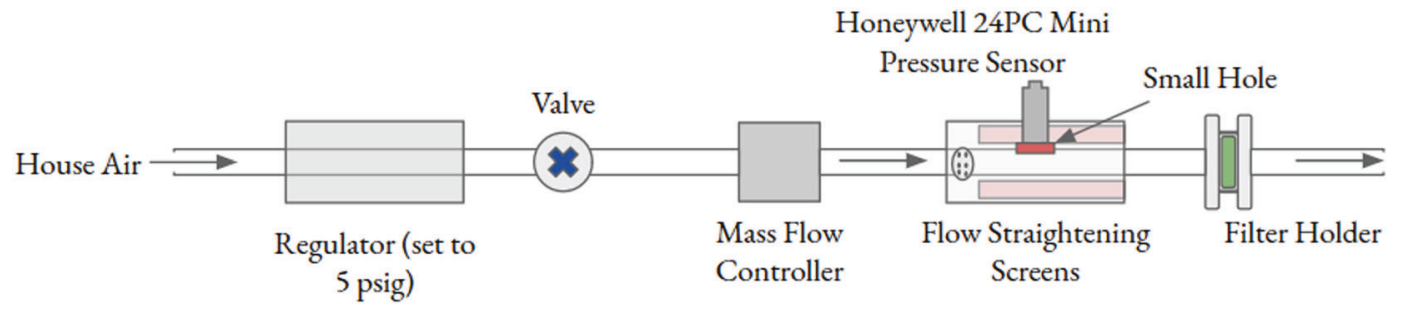

Fig. 2 Experimental setup to test flow impedance test for breathability. Based on ref. [32].

However, the analysis was done primarily with the AirNet to demonstrate that only a single reliable detector is critical for such an assessment. Downstream concentrations of the aerosols are checked regularly without a mask in place to confirm that they match those expected based on the source chamber concentrations and dilution rates. Periodic downstream measurements with the second SEARCH monitor were done to cross-check the observed filtration efficiencies, dilution rates, and aerosol transmission.

Downstream flow rates are controlled to change the face velocity used to test the filter material and provide the suction from the source chamber. The AirNet is controlled by a built-in orifice (2.8 LPM) and a second mass flow controller (AliCat) is used to pull the remainder of the flow through the system (e.g., 1.7 SPLM for a $10-\mathrm{cm} / \mathrm{s}$ face velocity), where vacuum is generated using two separate vacuum pumps. At any given face velocity, changing the dilution flow rate adjusts the test aerosol concentration. A more detailed description of the flow rates shown in Fig. 1 can be found in Section S1. The filtration efficiency for any of the size-resolved bins is defined as

Filtration efficiency[\%] $=\left(1-C_{\text {downstream }} / C_{\text {upstream }}\right) \times 100 \%$,

and for the purposes of this study was further substantiated by measuring across a series of dilution factors (with and without mask material in place) to gain multi-point measurements across a range of test aerosol concentrations at a given face velocity (see Section S4 and Fig. S6). Information related to the cost of equipment as well as criteria for optimizing concentration levels are described in Section S4.

\section{Flow impedance testing}

Flow impedance assessment of the mask materials is conducted to gauge the potential breathability of a given mask. Proper use of a mask mandates that the mask creates a good seal around one's face. This forces intake air to be limited to what can pass easily through the mask, which means that enough pressure is needed from breathing to drive the air flow. Regardless of filtration capability, a mask with low breathability still poses a risk to the wearer due to the difficulty of breathing normally.
The NIOSH procedure takes into account the effect of mask area on the maximum pressure allowed for a specified volume flow (the ratio defining an "extrinsic impedance") by using a full mask for the test. Here, we employed a straightforward apparatus to measure the intrinsic impedance of mask material by measuring the pressure drop (in $\mathrm{mm}$ $\mathrm{H}_{2} \mathrm{O}$ ) across a 40-mm disk of mask filter material as a function of the face velocity (calculated from the volume flow rate and the $40-\mathrm{mm}$ disk area of $12.6 \mathrm{~cm}^{2}$ ). The sample disk diameter here was determined by the size of a commercially available plastic filter holder, which was used for its easy availability, low cost, good sealing properties, and dual applicability in the rapid screening setup described below. The slope of the linear fit to these points is the intrinsic impedance of the material in units of $\mathrm{mm} \mathrm{H}_{2} \mathrm{O} /(\mathrm{cm} / \mathrm{s})$. The experimental apparatus (Fig. 2) is from Petculescu and Wilen [32] and further described in Section S3.

To get a value for the extrinsic impedance for a given mask, we divide the intrinsic impedance by the mask area, either measured directly or approximated based on mask type and geometry. It should be noted that the instrumentation used here could easily be replaced with low-cost commercial rotameters and manometers to measure flow and pressure.

\section{A rapid screening setup for testing aerosol filtration}

We also evaluated a rapid screening approach with the understanding that initial screening of large inventories is necessary to determine which masks/respirators warrant further testing and also that some communities and facilities may be constrained in terms of available instrumentation for aerosol measurement and flow control. This furthersimplified setup (Fig. 3) relies on a filter holder (same as that used for impedance) and a single AirNet 210 detector, with a fixed volume flow rate of $2.8 \mathrm{LPM}$, and configured to read out count data through its analog outputs. The face velocity through the filter was modified with a pair of lasercut ring inserts to test at $\sim 13 \mathrm{~cm} / \mathrm{s}$ (see images in Fig. S2). With this setup, adjusting the filter area to achieve the appropriate face velocity is critical, otherwise slow face velocities atypical of breathing conditions might skew results and give false positive results. 
(A)

Chamber

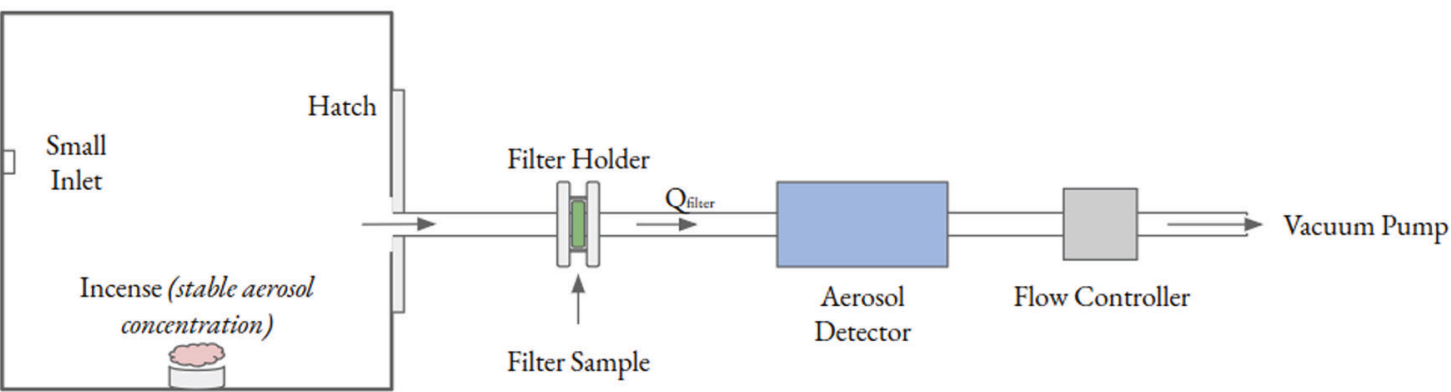

(B)

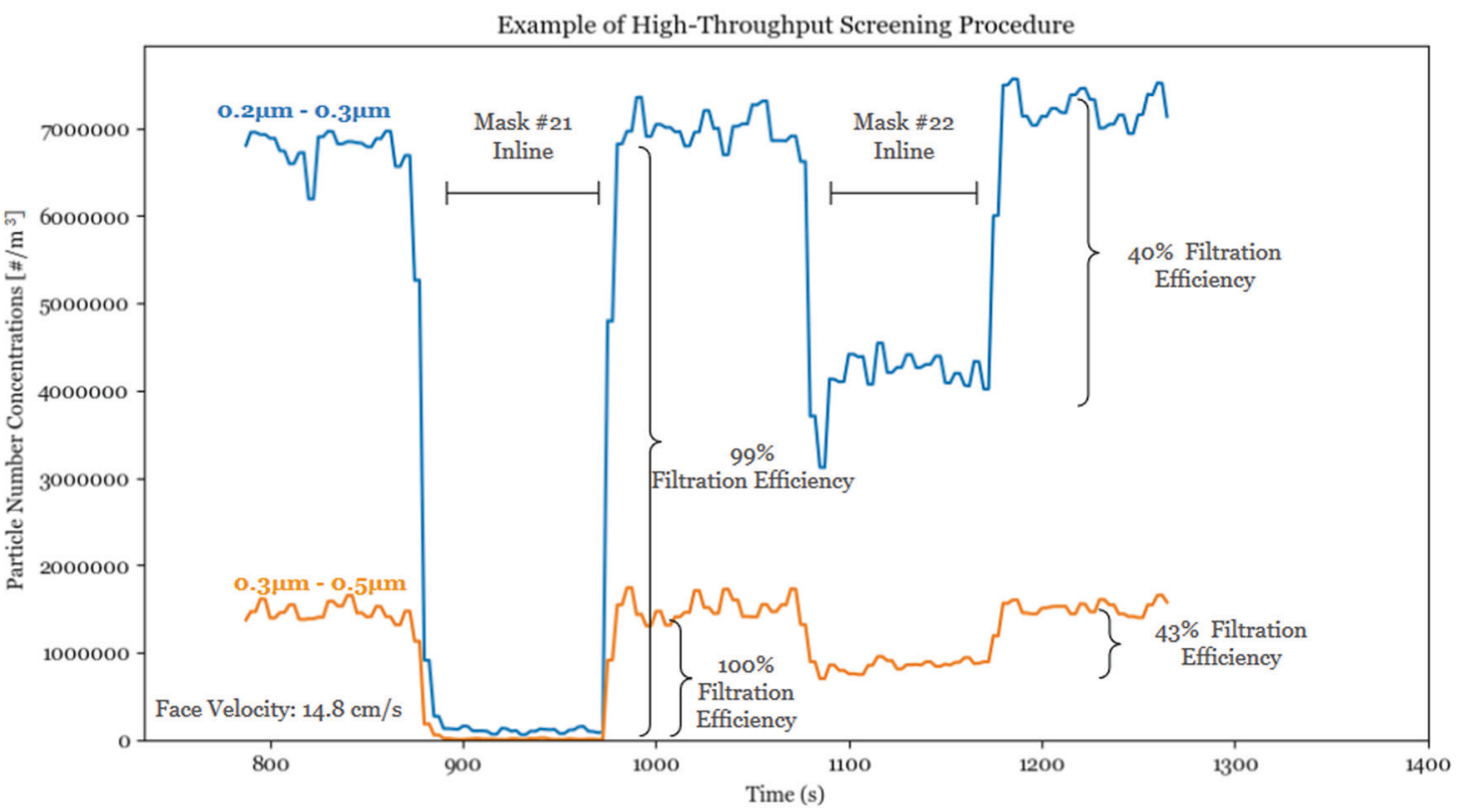

Fig. 3 A rapid screening setup. a Schematic and $\mathbf{b}$ example test procedure.

An example procedure is shown in Fig. $3 \mathrm{~b}$ where two mask materials are inserted and removed to assess against upstream concentrations without the mask in place. If ambient aerosol concentrations are sufficiently stable in the room where testing is occurring, ambient air can serve as the aerosol "source," as was the case for this evaluation approach. Data and results of this testing are described in supplemental information. Although a second AirNet 210 detector was used here, which allowed for a direct comparison to the full technique, any instrument that provides size-resolved aerosol concentration measurements could possibly work for this approach (see "Considerations" section).

\section{Results and discussion}

\section{Aerosol filtration efficiency}

While the instrumentation package measured an aerosol size range of $0.2-10 \mu \mathrm{m}$, aerosol filtration analysis focused on the range of $0.2-1 \mu \mathrm{m}$ for these reasons: this range is closer to sizes of interest in the NIOSH/FDA methods; it targets the most challenging aerosols to filter $(0.2-0.3 \mu \mathrm{m})$; and this particle diameter range is where mask performance differentiated most for the masks and instruments used in this study. A set of face velocities were examined to span typical inspiratory flow rates and typical test procedures [5], but primarily focused on $10 \mathrm{~cm} / \mathrm{s}$ for inventory screening (Fig. 4) as discussed earlier. Prior to recommending that a mask be used in service, multiple trials of the same mask type across a set of individual masks from a delivered lot are conducted to ensure consistent results in the data. Results from repeated tests of a single mask type (Fig. 4a inset) show variability within mask lots, which were larger for a poorly performing mask type (\#15), compared with the six N95 tests that ranged $98-99 \%$ at $10 \mathrm{~cm} / \mathrm{s}$. Repeat tests of the same exact mask sample on different days resulted in minimal variance and demonstrated consistency in the experimental setup. Specifically, the error was $0.7 \pm 0.6 \%$ in absolute deviation between tests (i.e., Eff.test $t-$ Eff.test $t+1_{1}$ ) for the $0.2-0.3 \mu \mathrm{m}$ size bin. 


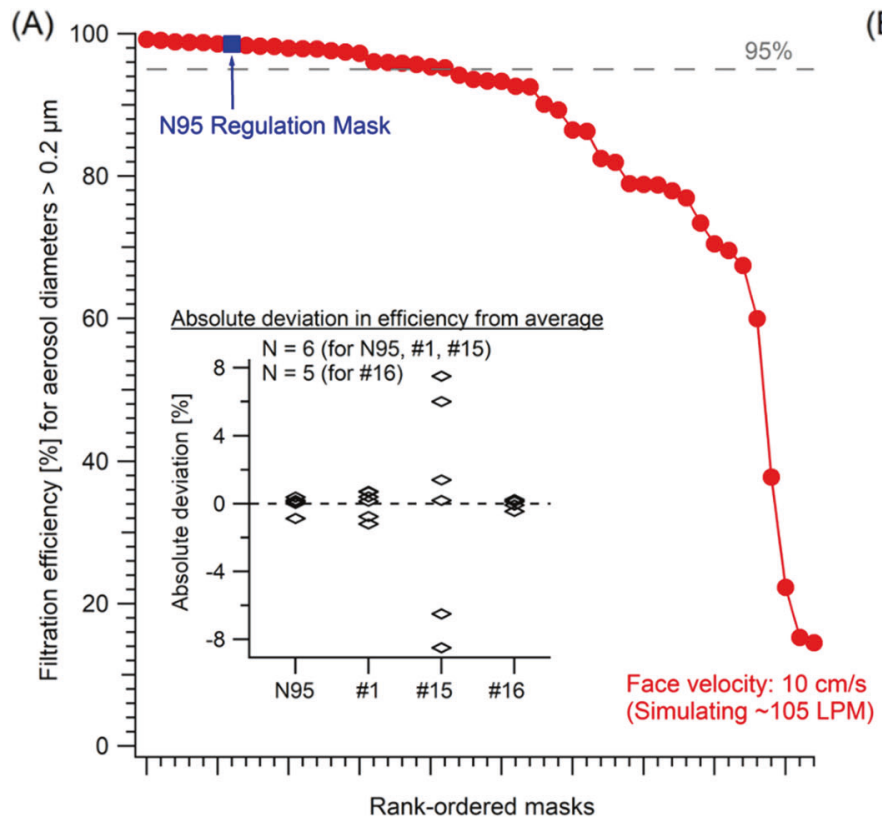

Fig. 4 Filtration efficiency of 47 commercially available masks. a Filtration efficiency for all aerosols of $0.2 \mu \mathrm{m}$ and larger, shown with a regulation-tested N95 mask (blue marker) at a face velocity of $10 \mathrm{~cm} / \mathrm{s}$, roughly simulating an 105 LPM inspiratory breathing rate; and $\mathbf{b}$ mask performance shown for three aerosol size fractions from 0.2 to $1 \mu \mathrm{m}$. Efficiencies shown are determined via aerosol number concentrations

As shown in Fig. 4, tests conducted on 47 nonregulation masks using this setup reveal that a number of commercially available masks perform similarly to the regulation N95 mask's aerosol filtration for $0.2 \mu \mathrm{m}$ and above. Then, there are a range of masks with relatively weaker filtration efficiencies (i.e., $80-95 \%$ for aerosols $>0.2 \mu \mathrm{m}$ ). Yet, a subset of commercially available masks have poor performance (i.e., $<80 \%$ ) relative to N95 or similar masks. It is important to note that the masks tested here focus on nonregulated, commercially available masks, most of which are purported to be efficacious for aerosol filtration at, or near, that of an N95 respirator (Table S1). The performance of the ten commercial (traditional) surgical-style masks tested varied widely $22-95 \%$ (see Table S1) with an average of $72 \pm 19 \%$. While materials for homemade masks could be tested with this setup, it was generally outside the scope of this PPE survey (the one homemade mask and one commercially made alternative mask donated to the hospital included in this study performed very poorly, i.e., $14 \%$ ).

There was significant disparity in the performance of masks purported to be N95 equivalent (i.e., KN95) or even labeled as "N95," spanning efficiencies of 38\% (Mask \#22) to $99 \%$ (see Table S1 for values). Some KN95 masks performed consistently well, but others did not. For example, Mask \#1 performed consistently well while Mask \#15 had significant variance (Fig. 4a inset) with two masks from \#15's lot performing at or near $95 \%$ filtration and others

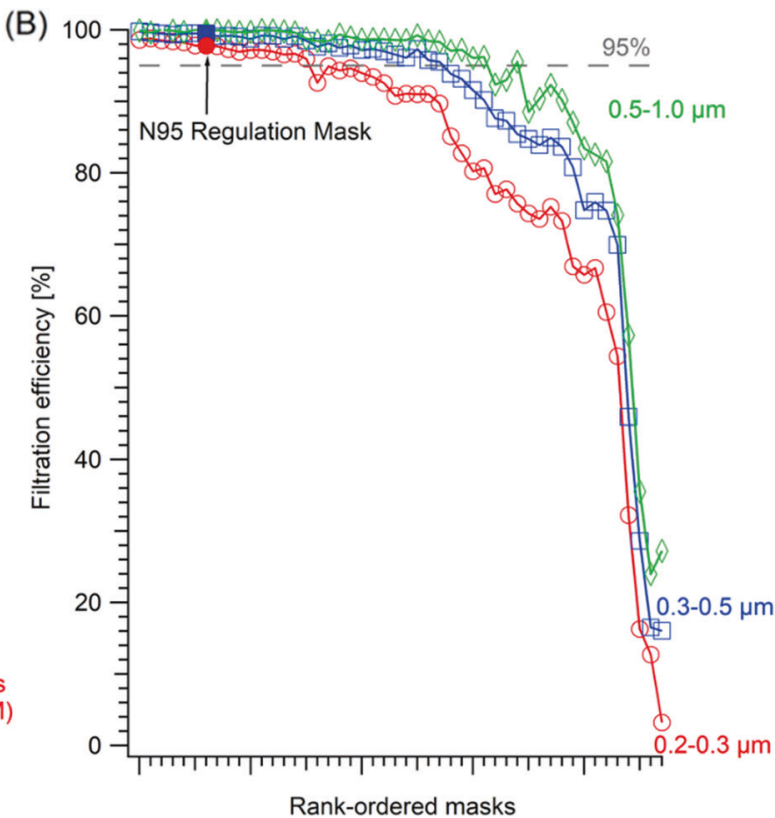

and the a inset shows variations across masks tested for four mask types, displayed as the absolute deviation in aerosol filtration efficiency from the average (i.e., Eff. Sample $i-$ Eff. $_{\text {Avg.). }}$. Mask numbers in the inset (and Fig. 5) refer to cataloging in Table S1 and not rankordered performance.

reaching as low as $78-82 \%$. These results raise the importance of both testing a significant sample size of masks from any received lots and the value of validation independent of vendor assurances.

Mask filtration efficiencies expectedly varied as a function of aerosol size. Given the higher efficiency of inertial impaction for larger aerosols with more mass and momentum, the masks generally performed better for larger aerosols (Fig. 5). While mask filtration performance can be similarly differentiated, absolute efficiencies are greater when considering all aerosols (i.e., $>0.2 \mu \mathrm{m}$ ) than solely aerosols of $0.2-0.3 \mu \mathrm{m}$ (Fig. 4, S7). Consistent with aerosol filtration theory, the removal of smaller aerosols is better at slower face velocities (Fig. 5) given the dependence of "diffusive" losses (i.e., Brownian motion to the filter fibers) on flow rates (i.e., timescales for air transport through the filters). This aerosol size dependence is important since SARS-CoV-2-containing aerosols are distributed across a wide size range and aerosols with diameters of $0.1-0.5 \mu \mathrm{m}$ can remain airborne longer [18, 19, 24, 25]. With regard to testing protocols, Fig. 5 demonstrates clearly how flow rate (i.e., face velocity) influences filtration efficiencies.

\section{Impedance and breathability}

Using the technique described above, the intrinsic impedance was measured and tabulated for the mask inventory 


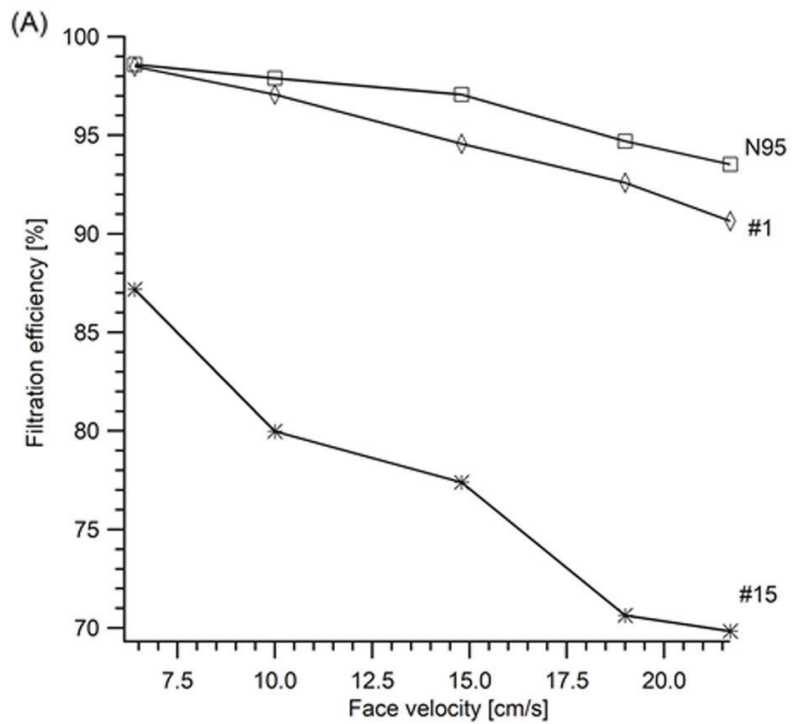

Fig. 5 Filtration efficiency as a function of face velocity resulting from typical breathing flow rates. a Aerosol removal efficiency $(>0.2 \mu \mathrm{m})$ of three masks: an N95 regulation mask and two masks
(B)

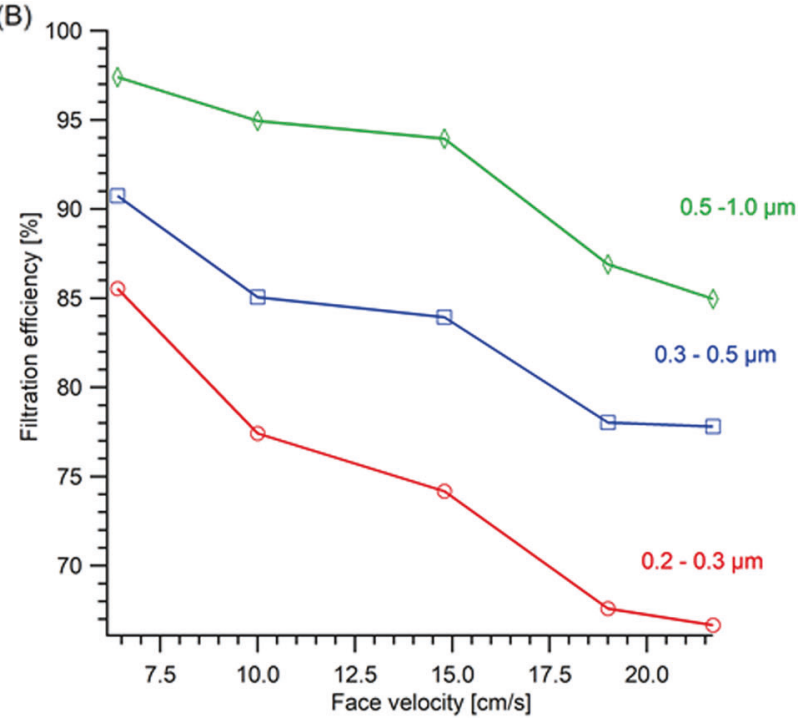

marketed as KN95 (\#1 and \#15). b Size-resolved removal efficiency for a moderately performing mask (\#15 from a).
Fig. 6 Measured impedance values shown against breathability index (i.e., N95 intrinsic impedance/intrinsic impedance) where all masks are within the NIOSH threshold for impedance. Points are colored based on filtration efficiency, and no correlation was observed between impedance and filtration efficiency (see Fig. S7a).

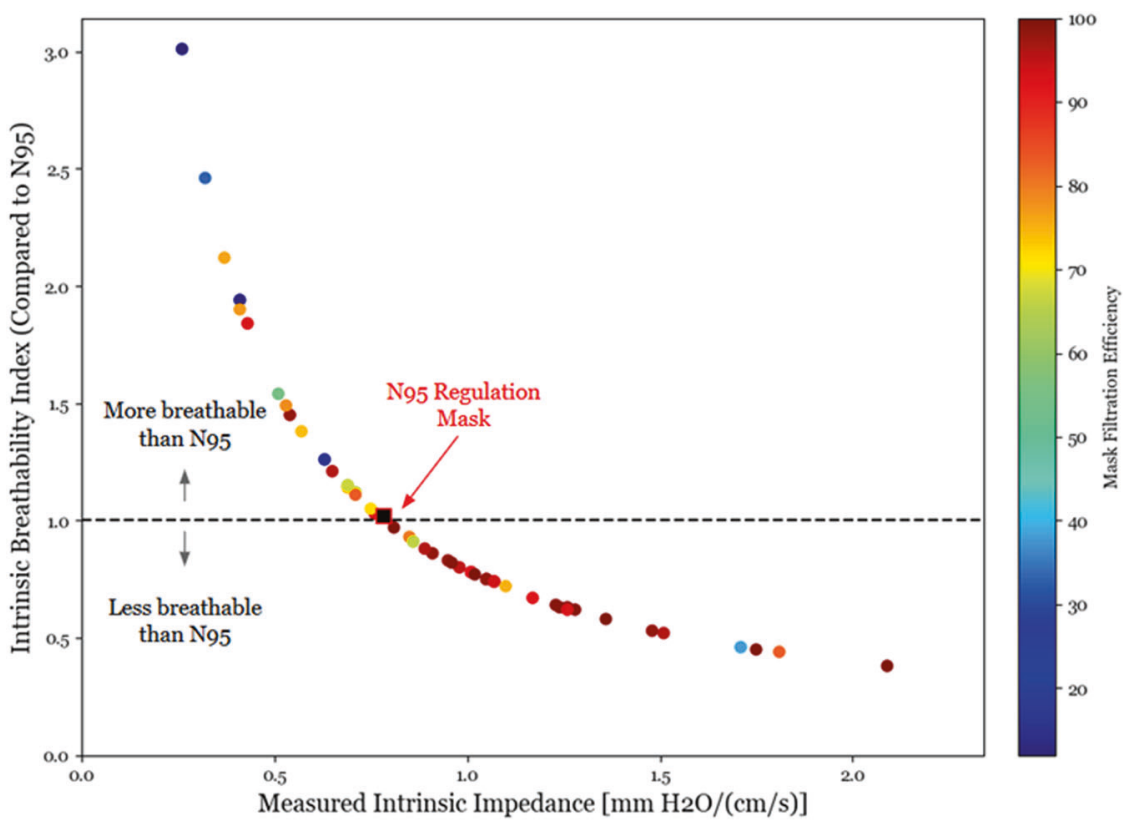

which is defined as the ratio of the intrinsic impedance for a mask compared with a standard N95 mask. In Section S4, we discuss various implications for the filtration, impedance, and face sealing relating to the mask area. Presented with a large array of masks to test, efforts to include area measurement were streamlined. Some masks were excluded from consideration early, based on poor filtration of the mask material. Masks found to be made of material with the highest measured filtration efficiencies were prioritized for more extensive examination. Among these, higher priority ison, we also present an "intrinsic breathability index" 


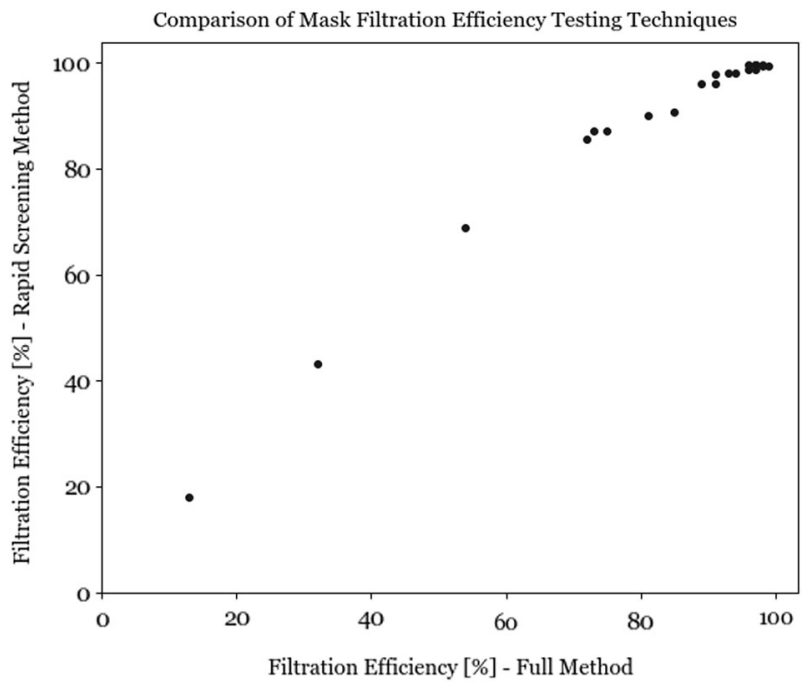

Fig. 7 Comparison of rapid screening setup (Fig. 3) compared with primary setup (Fig. 1) showing equivalent ranking of mask/respirator performance. Filtration efficiencies are shown for $0.2-0.3 \mu \mathrm{m}$ aerosols. Tested face velocities were not the same between the two approaches.

for use in hospitals was then given to masks with the smallest extrinsic impedance, also taking face sealing into account.

\section{Rapid screening results}

To evaluate the rapid screening setup (Fig. 3), we tested this approach for half of the masks tested in the Fig. 1 setup. The rapid screening approach's filtration efficiencies were consistent with those of the primary setup and effective for rank-ordering masks (Fig. 7). The differences in filtration efficiencies in Fig. 7 are not random, but well-correlated when fit to a power function (Fig. S13), potentially owing to the combined effects of differences in in-room aerosol size distributions and face velocities. Based on these results, we conclude that this method has efficacy as a rapid screening method, where 26 masks can be preliminarily screened in 80 min (Fig. S11). However, frequent cross-comparison to N95 benchmark masks is essential between operational sessions since variations in-room aerosol composition can affect results. More details about correlations between the two techniques and calibration procedures are described in the Section S5.

\section{Conclusions: considerations and best practices}

As defined above, these methods are not intended to replace regulation approaches, but to provide accessible screening approaches where necessary for emergency evaluation of incoming masks/respirators. The goal is to allow users to rank-order masks in comparison with N95 "benchmark" masks (in their possession) to enable more informed decisions and prioritization of PPE for use. Mask fit and durability must also be assessed. As mentioned earlier, a commercial leak detection apparatus is used to characterize penetration of aerosols at the edges of the masks, and these results are also factored into the decision/prioritization process. To facilitate replicate setups, we outline a selection of considerations, potential issues, and best practices with additional detail in Section S4. Given that all masks here performed sufficiently in terms of breathability (i.e., flow impedance) compared with the NIOSH threshold, our discussion in the main text is focused on filtration test considerations, where the tested masks performed more variably.

The primary testing setup described in Fig. 1 is not the sole feasible configuration, as is readily demonstrated with the rapid screening approach (Fig. 3). However, the primary setup does offer certain advantages that should be considered by future users: (a) consistent and stable spherical polydisperse aerosol populations that span the range of target aerosol sizes; (b) repeatable flow rates delivering the test aerosol mixture at atmospheric pressure and representative face velocities (e.g., $10 \mathrm{~cm} / \mathrm{s}$ ) that allow for discrimination of mask performance; (c) leak-checked systems, especially in the filter holder where bulky mask materials increase the potential for leaks (note: flow balance can be confirmed using multiple flow controllers; Fig. 1); (d) frequent confirmation of test aerosol concentrations via downstream instruments (with no filter in place) checked at all flow and dilution conditions used, with redundant instruments up- and downstream (if available); (e) regular checks of filtration efficiency against N95 "benchmark" masks; and (f) metal tubing and filter holder (all grounded) are best practice to avoid electrostatic aerosol losses, but nonmetallic components may work with frequent comparisons to "blank" measurements without a filter. Similarly, digital mass flow controllers are used in this study, but sufficiently precise rotameters or flow constrictions may prove effective substitutes.

Aerosol instrumentation is a critical consideration, and differences between available instrumentation are considerable across the global scientific and hospital community. As such, this study is carried out using a total aerosol instrumentation cost of under \$2000 USD, and found high utility in the AirNet aerosol particle counter used for clean-room monitoring. While multiple monitors are included in Fig. 1 and used in cross-checks, the conclusions were derived primarily with a single detector. The key elements are that (a) the aerosol concentrations used for testing must be adjusted based on the sensitivity of the instrumentation such that the signalto-noise ratio of the measurements are sufficiently high to determine the filtration efficiency with some precision (i.e., accurately measure changes between upstream and downstream concentrations) while also avoiding exceeding upper limits of detection or linearity; (b) real-time instruments allow 
for faster screening of inventories and prioritization of masks; (c) size-resolved measurements are better suited to discern differences in mask performance and more closely match aerosols in NIOSH/FDA tests since larger, easily filtered aerosols are major contributors to the mass distributions of combustion or in-room aerosols; and (d) it is beneficial to use aerosols that span the range of sizes of potentially viruscontaining aerosols $(>0.2 \mu \mathrm{m}$ in this setup), but smaller aerosols in this range are more challenging to filter at the typical face velocities and are preferentially tested in the NIOSH and FDA procedures. Yet, total aerosol number concentration measurements without size-resolution that include sizes below $0.1 \mu \mathrm{m}$ may skew efficiency results by incorporating filtration of combustion or in-room aerosols dominated by these smaller sizes, which are easily collected via Brownian motion, and are outside the size range of interest for SARS-CoV-2 aerosol transmission. While aerosol number concentrations were used to determine filtration efficiencies in this study, size-resolved mass concentrations could be used with appropriate test aerosol concentrations and instrument sensitivities.

Regardless of the testing setup, it is essential to screen a sufficient number of masks/respirators to constrain lot-to-lot variability (e.g., Fig. 4a inset) for masks that might be used in service. The NIOSH protocol requires 20 of 20 masks to exceed the $95 \%$ filtration efficiency. The exact number of randomly selected masks to be tested by an organization may depend on the supply of masks in a given lot and their likely application, but based on our results there are clear indicators of mask-to-mask variance within a sample size of five masks (Fig. 4a inset) that could be used to inform further examinations of variance.

Given that aerosol filtration efficiency varies with aerosol size, larger aerosols will be removed more effectively (i.e., $1-5 \mu \mathrm{m}$ ), but smaller aerosols (e.g., $0.2-0.5 \mu \mathrm{m}$ ) may provide more ability to discern between mask/respirator performance. Similarly, faster flow rates may provide more potential to discern between masks, yet care should be taken to not extend velocities outside the range of the typical conditions during breathing (coughing, speaking, etc.) that are examined in regulation testing (e.g., 3-14 cm/s) [5]. For a given flow rate, differences in face velocity with a full mask, compared with the subsections tested here, will vary with mask area, such that a larger surface area mask will have lower face velocities while in-use. Yet changes in filtration efficiencies for well-performing masks will be minor (e.g., with a decrease from 10 to $7.5 \mathrm{~cm} / \mathrm{s}$; Fig. 5a) and the face velocity can be adjusted to account for the mask area where necessary for verification.

The methods presented here rely upon regular comparisons to NIOSH N95 benchmark masks, as absolute filtration efficiencies will vary with changes in flow rates and aerosol sizes measured (e.g., Fig. 5). Similar aerosol concentrations and consistent flow conditions between masks must be maintained to minimize system variance that could affect data interpretation. Validation of a system should include testing a range of materials expected to have both excellent and poor filtration efficiencies. Finally, this approach does not convey the equivalent of an N95 certification, and this publication does not include a full comparison to NIOSH results other than the comparison with available N95-compliant masks.

Acknowledgements We would like to thank Dan Rosner (Yale), Jenna Ditto (Yale), Juan de la Mora (Yale), Jason Hancock (U. Conn.), Donal Sheets (U. Conn.), and Michael Plumley (U.S. Coast Guard Academy) for loaning equipment/instrumentation and/or helpful discussions. The SEARCH monitor used in this study was developed under Assistance Agreement No. RD835871 awarded by the U.S. Environmental Protection Agency to Yale University. It has not been formally reviewed by EPA. The views expressed in this document are solely those of the authors and do not necessarily reflect those of the Agency. EPA does not endorse any products or commercial services mentioned in this publication. CB is supported by the National Science Foundation Graduate Research Fellowship Program under Grant No. DGE1752134. Any opinions, findings, and conclusions or recommendations expressed in this material are those of the author(s) and do not necessarily reflect the views of the National Science Foundation.

\section{Compliance with ethical standards}

Conflict of interest DRG has externally funded projects on low-cost air quality monitoring technology (EPA, HKF Technology), which Yale has licensed to HKF Technology.

Publisher's note Springer Nature remains neutral with regard to jurisdictional claims in published maps and institutional affiliations.

\section{References}

1. Livingston E, Desai A, Berkwits M. Sourcing personal protective equipment during the COVID-19 pandemic. JAMA. 2020;323: 1912-4.

2. Lam SC, Suen LK, Cheung TC. Global risk to the community and clinical setting: flocking of fake masks and protective gears during the COVID-19 pandemic. Am J Infect Control. 2020;48:964-5.

3. Broadhurst R, Ball M, Jiang C. Availability of COVID-19 related products on Tor darknet markets. Statistical Bulletin no. 24. Canberra: Australian Institute of Criminology; 2020. p. 12.

4. Ippolito M, Iozzo P, Gregoretti C, Cortegiani A. Counterfeit filtering facepiece respirators are posing an additional risk to healthcare workers during COVID-19 pandemic. Am J Infect Control. 2020;48:853-8.

5. Rengasamy S, Shaffer R, Williams B, Smit S. A comparison of facemask and respirator filtration test methods. J Occup Environ Hyg. 2017;14:92-103.

6. Rengasamy S, Eimer B, Shaffer RE. Simple respiratory protectionevaluation of the filtration performance of cloth masks and common fabric materials against $20-1000 \mathrm{~nm}$ size particles. Ann Occup Hyg. 2010;54:789-98.

7. NIOSH. Filtering out Confusion: Frequently Asked Questions about Respiratory Protection, Fit Testing. Krah J, Shamblin M, Shaffer R (Eds). Pittsburgh, PA: U.S. Department of Health and Human Services, Centers for Disease Control and Prevention, National Institute for Occupational Safety and Health, DHHS 
(NIOSH). Publication No. 2018-129. 2018. https://doi.org/10. 26616/NIOSHPUB2018129.

8. NIOSH: Procedure No. TEB-APR-STP-0059, Revision 2.0. Determination of particulate filter efficiency level for N95 series filters against solid particulates for non-powered, airpurifying respirators standard testing procedure (STP). Pittsburgh, PA DHHS, Centers for Disease Control and Prevention, National Institute for Occupational Safety and Health, National Personal Protective Technology Laboratory. 2007. http://www.cdc.gov/ niosh/nppt1/stps/pdfs/TEB-APR-STP-0059.pdf.

9. FDA. Surgical masks - premarket notification $[510(\mathrm{~K})]$ submissions; Guidance for Industry and FDA staff. FDA. 2004. https://www.fda. gov/regulatory-information/search-fda-guidance-documents/surgicalmasks-premarket-notification-510k-submissions.

10. ASTM. ASTM F2299-03. Standard test method for determining the initial efficiency of materials used in medical face masks to penetration by particulates using latex spheres. West Conshohocken, PA: ASTM International; 2003.

11. ASTM. F2101-01. Standard test method for evaluating the bacterial filtration efficiency (BFE) of medical face mask materials, using a biological aerosol of Staphylococcus aereus. ASTM Stand. (F2101-01). 2001. p. 1553-7.

12. ASTM. F2100. Standard specification for performance of materials used in medical face masks. Ann. Book. Philadelphia, PA: ASTM Standards; 2011. p. 447-9.

13. Lindsley WG, Pearce TA, Hudnall JB, Davis KA, Davis SM, Fisher MA, et al. Quantity and size distribution of coughgenerated aerosol particles produced by influenza patients during and after illness. J Occup Environ Hyg. 2012;9:443-9.

14. Milton DK, Fabian MP, Cowling BJ, Grantham ML, McDevitt JJ. Influenza virus aerosols in human exhaled breath: particle size, culturability, and effect of surgical masks. PLoS Pathog. 2013;9: e1003205

15. Morawska LJ, Johnson GR, Ristovski ZD, Hargreaves M, Mengersen K, Corbett S, et al. Size distribution and sites of origin of droplets expelled from the human respiratory tract during expiratory activities. J Aerosol Sci. 2009;40:256-69.

16. Papineni RS, Rosenthal FS. The size distribution of droplets in the exhaled breath of healthy human subjects. J Aerosol Med. 1997; 10:105-16.

17. Xie X, Li Y, Sun H, Liu L. Exhaled droplets due to talking and coughing. J R Soc Interface. 2009;6 (Suppl 6):S703-14.

18. Liu Y, Ning Z, Chen Y, Guo M, Liu Y, Gali NK, et al. Aerodynamic characteristics and RNA concentration of SARS-CoV-2 aerosol in Wuhan hospitals during COVID-19 outbreak. 2020. https://doi.org/10.1101/2020.03.08.982637.
19. Chia PY, Coleman KK, Tan YK, Ong SW, Gum M, Lau SK, et al. Detection of air and surface contamination by severe acute respiratory syndrome coronavirus 2 (SARS-CoV-2) in hospital rooms of infected patients. 2020. https://doi.org/10.1101/2020.03.29.20046557.

20. Mikhailov E, Vlasenko S, Niessner R, Pöschl U. Interaction of aerosol particles composed of protein and salts with water vapor: hygroscopic growth and microstructural rearrangement. Atmos. Chem. Phys. 2003;3:4755-832.

21. Yang W, Marr LC. Dynamics of airborne influenza A viruses indoors and dependence on humidity. PloS One. 2011;6:e21481.

22. Goldsmith CS, Tatti KM, Ksiazek TG, Rollin PE, Comer JA, Lee WW, et al. Ultrastructural characterization of SARS coronavirus. Emerg Infect Dis. 2004;10:320.

23. World Health Organization. Modes of transmission of virus causing COVID-19: implications for IPC precaution recommendations. Scientific brief. World Health Organization; 2020.

24. Nazaroff WW. Indoor particle dynamics. Indoor Air. 2004;14 (Suppl 7):175-83.

25. Lai AC, Nazaroff WW. Modeling indoor particle deposition from turbulent flow onto smooth surfaces. J Aerosol Sci. 2000;31:463-76.

26. van Doremalen N, Bushmaker T, Morris DH, Holbrook MG, Gamble A, Williamson BN, et al. Aerosol and surface stability of SARS-CoV-2 as compared with SARS-CoV-1. N Engl J Med. 2020;382:1564-7.

27. Santarpia JL, Rivera DN, Herrera V, Morwitzer MJ, Creager H, Santarpia GW, et al. Transmission potential of SARS-CoV-2 in viral shedding observed at the University of Nebraska Medical Center. 2020. https://doi.org/10.1101/2020.03.23.20039446.

28. Jetter JJ, Guo Z, McBrian JA, Flynn MR. Characterization of emissions from burning incense. Sci Total Environ. 2002;295:51-67.

29. Wang B, Lee SC, Ho KF. Chemical composition of fine particles from incense burning in a large environmental chamber. Atmos Environ. 2006;40:7858-68.

30. Lin TC, Krishnaswamy G, Chi DS. Incense smoke: clinical, structural and molecular effects on airway disease. Clin Mol Allergy. 2008;6:3.

31. Levy Zamora M, Xiong F, Gentner D, Kerkez B, Kohrman-Glaser J, Koehler K. Field and laboratory evaluations of the low-cost plantower particulate matter sensor. Environ Sci Technol. 2018; 53:838-49.

32. Petculescu A, Wilen LA. Oscillatory flow in jet pumps: nonlinear effects and minor losses. J Acoustical Soc Am. 2003;113:1282-92.

33. NIOSH Procedure No. TEB-APR-STP-0007. Determination of inhalation resistance test, air purifying respirators standard testing procedure (STP). https://www.cdc.gov/niosh/npptl/stps/pdfs/TEBAPR-STP-0007-508.pdf. 